\title{
Developing Digital Musical Instrument, mPoi, by Taking Advantage of Sensor Interfaces: Focused on the Multimedia Music Piece, "Jwibul"
}

\author{
Sangbong $\mathrm{Nam}^{1}$ ), Donoung Lee²)
}

\begin{abstract}
This article describes the mPoi, a sensor-based digital musical instrument, and how its music is composed. It is a unique interactive electronic musical instrument playing sound and light, which is converted from the trajectory of circular-based motion drawn by the performance and the momentum of the mPoi instrument. This paper explores the process of how mPoi is used in converging various art forms. It presents the concepts and the motivation of the mPoi project, the design and construction of the mPoi instrument, the methodologies on mapping information from sensors to music, an analysis of "Jwibul" that was composed for mPoi, and lastly, the future development plans for the said instrument.
\end{abstract}

Keywords: Wireless Sensor Interface, Sensor-based Instrument, mPoi, Circular Motion, Electronic Musical Instrument

\section{Introduction}

A musical instrument can be considered as a device that translates the performer's gesture into music. The Inexpensive sensing technologies and programmable microcontrollers[1][2] have enabled the expansion of the range of performer's movements that can be converted to music. Various types of musical instruments that utilize motion sensors embedded in smart devices, game controllers and other sensors interfaced with personal computers were designed over the last several years[3-7]. Although signal and physical models have already been considered sufficiently mature to be used in a concert situation[8], there are ongoing researches on input device technologies capturing the different human gestures.

The mPoi project is a case study to explore and develop an entirely new form of art using

Received(March 04, 2020), Review Result(1st: April 02, 2020, 2nd: May 25, 2020), Accepted(June 25, 2020)

1) (Researcher) 08862 Center for Arts \& Technologies at Seoul National University, Seoul National University, 1 Gwanak-ro, Gwanak-gu, Seoul 151-742 Korea

email: sangbongnam@gmail.com

2) (Professor, Corresponding Author) 08862 Center for Arts \& Technologies at Seoul National University, Seoul National University, 1 Gwanak-ro, Gwanak-gu, Seoul 151-742 Korea email: dolee@snu.ac.kr 
Developing Digital Musical Instrument, mPoi, by Taking Advantage of Sensor Interfaces: Focused on the Multimedia Music Piece, "Jwibul"

an input device technology. New form means that information from performer's gesture is combined organically with multimedia such as music, dance, video, and light to create artistic results. It also makes use of existing art forms. The mPoi, short for musical poi, utilize the poi performance that originated from Maori people in New Zealand. In the performance, poi dancers swing tethered weights in a variety of rhythmical and geometric patterns[9].

The initial motivation for the mPoi project is Jwibulnori, a traditional Korean play. To play Jwibulnori, people twirl a metal container filled with burning wood and tied to a strong string during the night of full moon. Swinging an object is a positively entertaining experience for men and women of all ages. This idea developed further along with the poi performance The poi performance not only has the basic performance of swinging objects similar to Jwibulnori, but also has value as an art performance through its diverse patterned variations. The various geometric influence can be easily discerned in contemporary poi[10], and more people enjoy this as part of juggling or flow art in many festivals around young people. In the mPoi project, sound was added to this experience. The sound produced with the friction of circular motions in the air expands into music with the power of digital technology. As an extension of the body and its movement, the mPoi utilizes the creative performance of Poi to generate sound and music.

\section{Prototyping mPoi}

\subsection{Construction of the mPoi}

The construction of the mPoi is based on the general shape of a poi: a weight attached to the end of flexible cord[11]. A performer holds the instrument with his or her hands and swings around in circular patterns. Thus the instrument moves as an extension of the player's gesture and movement.

The performer swings the mpoi in a controlled manner and interacts with it to make unique sounds. A performer can usually play with one or two mPois as illustrated in [Fig. 1].

The mpoi instrument uses an $\mathrm{x}$-OSC as a sensor interface. The $\mathrm{x}$-OSC is a wireless I/O board connected to a gyroscope sensor and an accelerometer sensor to interface electronics to any software using OSC[12]. Integrations of the data from sensors calculate the velocity and position of the object. The information from the sensor is sent to the computer using wireless 
network, then translated to the sound using the main speakers in a concert hall[13]. [Fig. 2] introduces the system of the instrument.

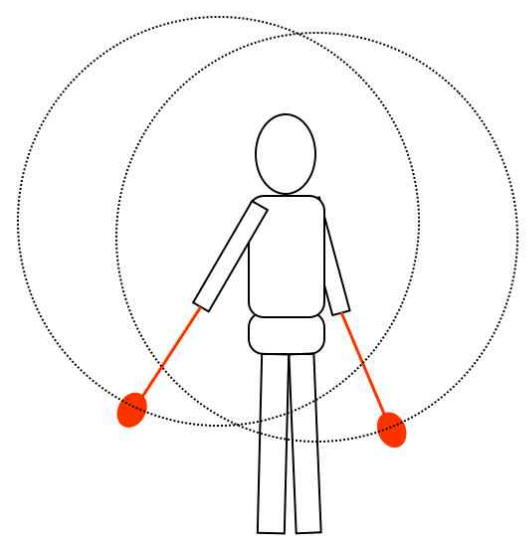

[Fig. 1] Illustration of the mPoi Performance

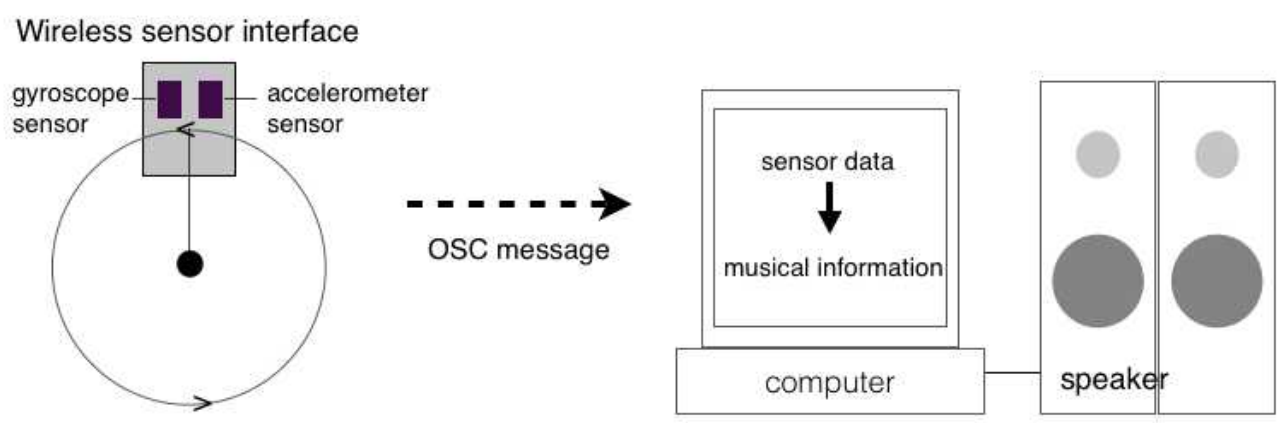

[Fig. 2] The Schematic of the mPoi

\subsection{Mapping Data from Sensors to Sound}

\subsubsection{Collecting Data from Sensors}

A gyroscope sensor and an accelerometer sensor were used to track down the kinetic value of a circular motion object, as shown in [Fig. 3]. The horizontal axis represents time and the longitudinal axis represents the amount of kinetic volume. The variables $x, y$, and $z$ are the three axes of the sensors. For a gyroscope sensor, the $x$-axis and $z$-axis are raised and lowered at a constant rate, whereas the $\mathrm{y}$-axis stays at positive values for clockwise movements and negative values for counter-clockwise movements. Although the accelerometer sensor is irregular compared to the gyroscope sensor, the x-axis and z-axis can repeatedly be seen rising and falling with its own pattern. 
Developing Digital Musical Instrument, mPoi, by Taking Advantage of Sensor Interfaces: Focused on the Multimedia Music Piece, "Jwibul"

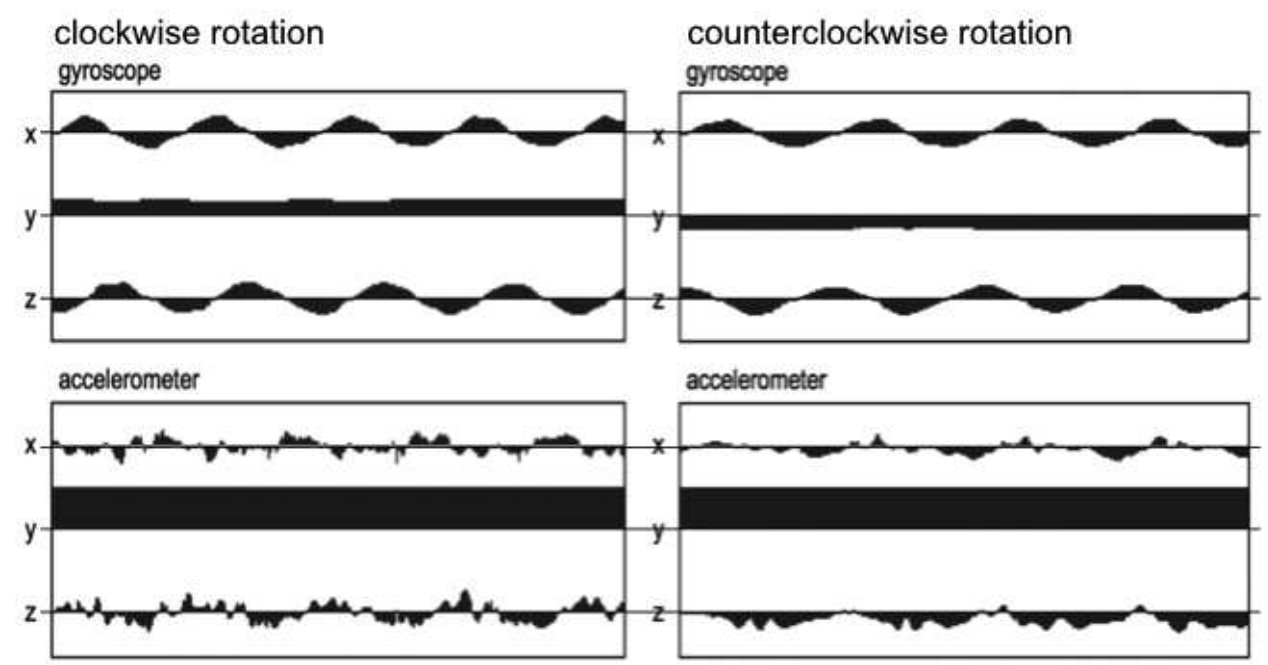

[Fig. 3] Results of Triple Axis from a Gyroscope Sensor and Accelerometer Sensor

\subsubsection{Mapping Data to Sound}

The sensor values transmitted from the wireless sensor interface can be expressed in a musical language through various mapping methods. In particular, the values of the $\mathrm{x}$-axis and z-axis, which continue to be raised and lowered at a constant rate, can be easily compared with the low frequency oscillator (LFO) used in music. These values can be applied to change musical variables such as volume, pitch, or timbre. First, when the sensor values are substituted for the volume values, there is a louder sound as the speed of the circular motion increases further. Second, when the sensor values are substituted for the pitch values, there is a higher pitch sound as the speed of the circular motion increases. Finally, when sensor values are substituted for parameters of various sound effects including the component filters, the timbre changes with the speed of the circle movement.

\section{Performing mPoi}

\subsection{Patterns of Poi Performance}

The mPoi uses the Poi performance actively to convert the various patterns of the instrument's moving trajectories into varying sounds. A poi performer usually swings two pois in various tricks such as weaves, butterflies, wraps, flowers, contact poi, buzzsaw, stalls, isolations, extensions and hyperloops. The audience sees geometric shapes with the trajectories of these 
patterns. They also hear the sound these trajectories produce.

\section{2 "Jwibul" for mPoi and Electronic Music}

"Jwibul" is a multimedia piece for mPoi and electronic music. The various patterns shown in the Poi performance were mapped to fit the structure of the work and maximized with artistic results. The trajectory information from the wireless sensor interface attached to the instrument is transmitted to the computer by OSC massage and allows real-time control of audio, video and LEDs using Max, a visual programming language by Cyling74. This paper focused on audio among various media, analyzing how this instrument was used throughout the work for new artistic expression.

Jwibul is about six minutes long, with the structure of the piece largely divided into three sections of $\mathrm{ABA}^{\prime}$ design, consisting of five sections including the intro and the outro as shown in [Fig. 4]. The instrumentation of the piece is largely divided into two parts - the mPoi of the melodic role and an accompaniment based on electronic music.

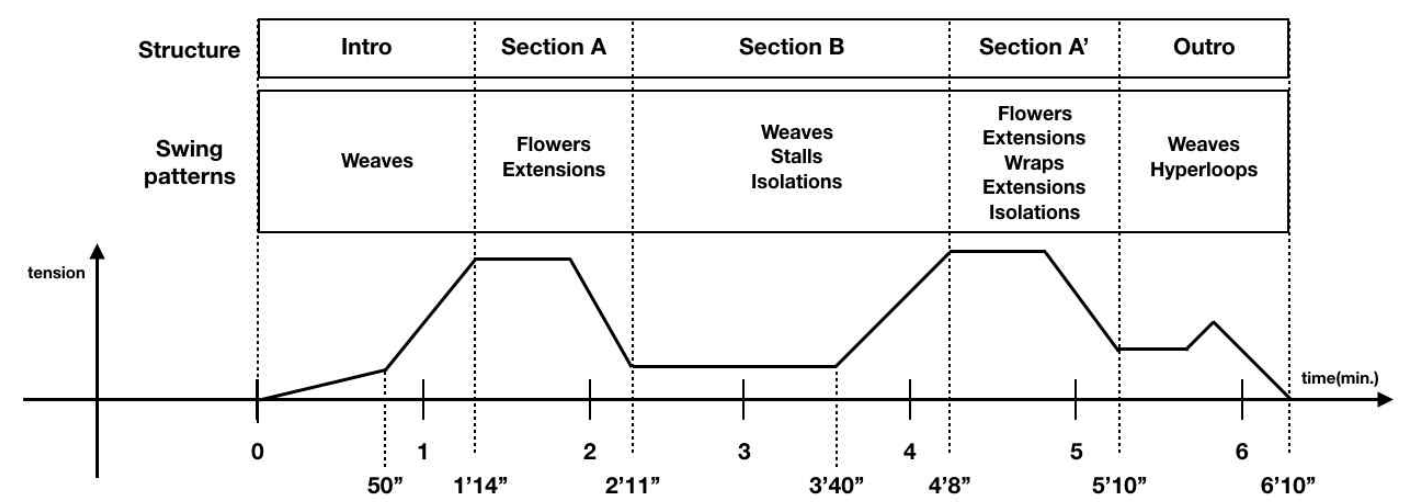

[Fig. 4] Structure and Swing Patterns of "Jwibul"

\subsubsection{Intro}

The goal of the introduction of the Jwibul is to make the audience aware that the mPoi's movements are expressed in real-time with sound and light. Since the mPoi is an instrument with an entirely new concept, giving the audience its background at the beginning of a piece played is very effective in understanding the music.

At first, the accompaniment was minimized at the beginning and focused on the sound of the instrument's movement, making it possible to express more intuitive sounds as it moves. In 
Developing Digital Musical Instrument, mPoi, by Taking Advantage of Sensor Interfaces: Focused on the Multimedia Music Piece, "Jwibul"

addition, the values from the accelerometer sensor are mapped to the sound of the instrument so that it can react sensitively even with a slight movement of the angle. The timbre of the instrument was based on a modified sign wave, which was designed to be more scratched than the sound of a pure sign wave, allowing the movement of the instrument to be more intuitive. The performer starts with a little movement with the instrument in both hands and gradually changes into a weaves pattern. The additional background sound comes in to build up the musical tension and moves on to the next part - section A - with the tension reaching its highest level.

\subsubsection{Section A}

Section $\mathrm{A}$ is the part where the tension of the piece explodes, in which the performer uses the pattern of flowers, the most colorful of the Poi patterns, to make a powerful sound in high range. Afterwards, in areas where tension decreases, the performer uses the pattern of extensions and finishes the section by holding the two mPois by hands.

\subsubsection{Section $B$}

Section B corresponds to a slow section of fast-slow-fast tempo, a traditional musical form, and has a slower tempo and lower tension than section A. The timbre of the instrument is based on the continuous repetition of the short notes of the percussion sound. The performer uses patterns of weaves, stalls and isolations appropriately and changes the swing speed, which is mapped to the adjustment of the instrument's timbre and volume to keep the quiet tension constant throughout the section. The sound of building up the musical tension is then started, as in intro section, and then moved on to the next part - section $\mathrm{A}^{\prime}$ - with the tension reaching another highest level.

\subsubsection{Section $A^{\prime}$}

Section $A^{\prime}$ is a section where the tension of the piece explodes again, and the performer uses a trick with a pattern similar to section A but more colorful. Not only the pattern of flowers, but all the patterns shown in section A, including raps, extensions and isolation, are reconstructed to make the climax of the performance.

\subsubsection{Outro}

In Outro, the previously explosive tension gradually decreases and finishes the performance. The performer uses the pattern of hyperloops just before the end to make a final twist using a 
certain amount of time in the highest-pitched sound. As the movement gradually decreases, the instrument stops and the music ends.

\subsection{Performance of "Jwibul"}

"Jwibul" for mPoi and electronic music has been performed in many places, such as "Fine Particle::Vibration 2018", "International Computer Music Conference 2018", "Hangang Fire Festival 2018", "2016 The Memory and Expectation of Sound", "2016 Ra Fire", "2015 WESA Festival", "2015 Seoul International Computer Music Festival", "2015 One month Festival”, "2015 Night::Yinshi" in Seoul, and "Musicacoustica-Beijing 2017" in China. [Fig. 5] shows the still cuts from the performance.

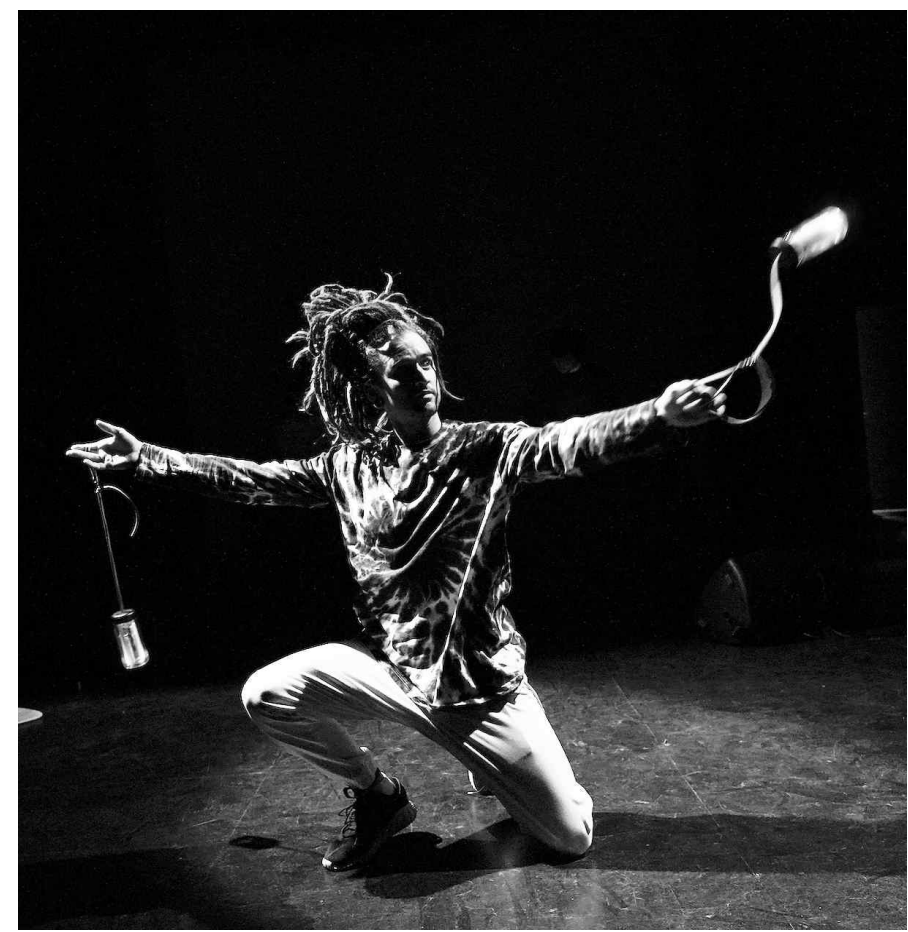

[Fig. 5] Still Cuts from the Performance "Fine Particle::Vibration"

\section{Conclusions and Future Work}

The mPoi is a novel electronic musical instrument utilizing technologies of the gesture tracking to create immersive and spiritual sound inspired by the Jwibulnori and Poi performances. It 
Developing Digital Musical Instrument, mPai, by Taking Advantage of Sensor Interfaces: Focused on the Multimedia Music Piece, "Jwibul"

has been developed since 2012 and has been performed in many places. In the near future, machine learning can be considered to analyze data from tracking the paths of the mPoi. Although there is a common circular motion in the movement of the mpoi, every movement is different from the other. This gives the performers difficulty in controlling their instrument. Machine learning could be helpful in sorting out the patterns more elaborately from the tracking information, yielding more possibilities for varied interesting sounds.

\section{Acknowledgement}

This work was supported by the Ministry of Education of the Republic of Korea and the National Research Foundation of Korea (NRF- 2016S1A5B5A01022364)

\section{References}

[1] C. B. Medeiros, M. Wanderley, Comprehensive review of sensors and instrumentation methods in devices for musical expression, Sensors, (2014), Vol.14, No.8, pp.13556-13591.

[2] J. Sullivan, A. Tibbitts, B. Gatinet, M. Wanderley, Gestural Control of Augmented Instrumental Performance: A casestudy of the concert harp in moco, The $5^{\text {th }}$ International Conference on Movement and Computing, (2018), June 28-30; Genoa, Italy.

[3] D. Ogborn, Composing for a Networked, Pulse-Based, Laptop Orchestra, Organised Sound, (2012), Vol.17, No.1, pp.56-61.

[4] H. J. Lee, S. Kim, Wii Arts: creating collaborative art experience with Wii Remote interaction, The $2^{\text {nd }}$ International Conference on Tangible and Embedded Interaction, (2008) February 18-20; Bonn, Germany, pp.33-36.

[5] A. McPherson, F. Morreale, Harrison, Musical Instruments for Novices: Comparing NIME, HCI and Crowdfunding Approaches, New Directions in Music and Human-Computer Interaction, German: Springer, Cham, (2019), pp.179-212.

[6] M. Wanderley, Gestural Control of Music, The International Workshop of Human Supervision and Control in Engineering and Music, (2001), September 21-24; Kassel, Germany, pp.632-644.

[7] F. Bevilacqua, N. H. Rasamimanana, E. Fléty, S. Lemouton, Baschet, The augmented violin project: research, composition and performance report, The $6^{\text {th }}$ International Conference on New Interfaces for Musical Expression (NIME 06), (2006), Jun 4-8; Paris, France, pp.402-406.

[8] Poli, Giovanni De, Sarti, Augusto, Borin, Gianpaolo, Musical Signal Synthesis, Musical Signal Processing, Netherlands: Lisse: Swets \& Zeitlinger, (1997), pp.5-30.

[9] K. Paringatai, Poia atu taku poi: Unearthing the knowledge of the past, Junctures: The Journal for Thematic 
Dialogue, (4: Movement) (2005), No.4, pp.23-34, tp://hdl.handle.net/10523/5131

[10] R. Walker, Poi Poi Revolution - A real-time feedback training system for object manipulation, Human Interface Technology Laboratory New Zealand, niversity of Canterbury, Master's Thesis, (2013)

[11] S. Nam, J. Kim, B. Martinson, M. Helmuth, Musical Poi(mPoi), The International Conference on New Interfaces for Musical Expression, (2013), May 27-30; Daejeon, Korea, pp.148-151.

[12] http://x-io.co.uk/x-osc, Feb 2 (2020)

[13] S. Nam, D. Lee, Exploring musical expression of circular motion by taking advantage of wireless sensor interfaces, Proceedings of HCI KOREA, (2017), Feburary 8-10; Gangwon, Korea, pp.253-256. 